\title{
Choice of Business Model and Pricing Strategy with Ad-Sponsor and Subscription Fee
}

\author{
Zhihui Wu \\ School of Business \\ East China University of Science and Technology \\ Shanghai, China \\ sophie_ne@163.com
}

\author{
Gang Liu \\ School of Business \\ East China University of Science and Technology \\ Shanghai, China \\ liugang@ecust.edu.cn
}

\begin{abstract}
This paper studies the strategy and best pricing tactics of two-sided platforms, and analyzes how the platforms will perform when facing both ad-sponsors and subscription fee.Researchers Researchers focus on the video websites as the research object, due to the peculiarity and negative cross-group network externalities of video websites. Based on the revenue structure and operation model, this paper considers 3 business models, including pure adsponsored model, pure subscription model, and a mixed model. With the help of modeling and game theory, researchers get the equilibriums of these three models. The result shows that, the pure model will incur a fixed portion of audience, if the platform wants to expand its customer, it needs the mixed model. Meanwhile, success of the mixed model depends on the add-on utility gained by VIP users compared with the free uses. The findings of this paper could contribute to development of video websites during the early and mid in their lifecycle.
\end{abstract}

Keywords-Business Model; Ad-sponsor; Model; Pricing; Video Researchers bsite

\section{INTRODUCTION}

Watch videos online has become an important way of entertainment, the "Internet network video application study in China of 2013" reported by CNNIC pointed out that, till the end of 2013, online video audiences are up to 428 million, and the usage rate of online video has reached $69.3 \%$. Online videos are internet applications providing video watching service with the aid of browser or client player software tools through the Internet. Video websites are operators of online videos; they are Internet Content Providers (ICP) as well as Internet Service Providers (ISP). Since the first video website was born in 2005, the online video industry has been developed in China for more than 10 years; the structure of this industry has undergone tremendous changes.

The revenue of video websites mainly comes from the following four aspects: income from the advertisers, subscription fee, distribution fee, and other value-add incomes. At present, advertising fee is still the main income of video websites in China, Advertising market accounted for the $58.2 \%$ proportion of the overall market size $^{[1]}$.

Most websites choose an appropriate business model according to their own resource superiority, audience distribution, as competing environment. Generally, video websites could choose one of the following three business model, the first one is ad-sponsored model, under this model, and audience could watch video with advertisements online with no charge. Most video websites adopted this model when first coming into the market and the famous video provider Hulu still sticks to this model. The second one is subscription model, websites adopting this model are able to provide highly differentiated video contents with high quality, most typical website of this model is Netflix, which was a video rental broker, and then transformed to an online video provider. In addition, the last one is a mixed model, websites under this model will provide audiences two kinds of products, one is free with advertisements and another one is based on subscription. At present, most web portals in China tend to choose the last model, such as Sohu Video and iQiYi.

This paper view the video website providing integrated contents as a two-sided platform that connects audiences and advertisers, and when the website chooses to adopt the subscription model, this two-sided platform degrades into one-sided platform. Rochet et al. (2003) ${ }^{[2]}$ built a basic model framework for two-sided platforms, and they thought the best prices that the platform set for actors from two sides depend on their price elasticity of demand. The platform will charge less to the side with higher price elasticity. Since the business models and competing strategies are quite different among different industries, recently some scholars study a particular industry and analyze best pricing under this circumstance. $\mathrm{Xu}$ et al. (2009) ${ }^{[3]}$, Casadesus-Masanell (2011) ${ }^{[4]}$, and Yong Chao et al. (20013) ${ }^{[5]}$ respectively studied the payment cards, software producers and video games, and gave the best pricing and competing strategies in these industries. This paper focuses on online websites, they are typical twosided platforms, i.e. the websites providing audience video contents and the ad-sponsors advertising service, and there exists cross-group network externalities between the audience side and the advertiser side. However, video websites are different from the traditional two-sided platforms; the advertisers will gain more profits if the platform could attract more audience, but the audiences dislike the advertisements embedded into the video contents. Therefore, the network externalities from audiences to advertisers are positive, and the network externalities from advertisers to audiences are negative.

Existing researches about ad-sponsored business model are concentrated on the fields of television programs and newspapers. Bourreau (2003) ${ }^{[6]}$ analyzed the trade-off between imitation strategy and counter programming strategy under the subscription based model and adsponsored model in TV industry. Prasad et al. (2003) ${ }^{[7]}$ considered the trade-off between advertisement fee and 
subscription fee of media providers, and they found that with the advertisers' willing to pay added, the monopoly platform would lower the subscription fee of the other side. Crampes et al (2009) ${ }^{[8]}$ studied the entry of media firms, and they found that without the entry threshold, there would be too many firms in the markets pervade with adsponsored actors. Casadesus-Masanell and Zhu (2010) ${ }^{[9]}$ viewed the newspapers as research object, allowing the incumbent to change its current business model; they studied the best competing strategy of the incumbent when facing the entrants adopting ad-sponsored business model. $\mathrm{Wu}(2014)^{[10]}$ focused the newspaper, and pointed out that if the firms chose to set price for advertisers according to the false issuance based on cross-group network externalities, the companies creating advisements would get hurt, and so did the social general welfare.

The operation modes of video websites are quite different from the modes of TV programs and newspapers, audiences could not only remove the ads, but also could watch exclusive videos, gain high-quality and independent broadband access by paying the subscription fee. Therefore, in the model of video websites, add-on utility gained by VIP users through paying is the key element. This paper concentrates on video website, and analyzes how a platform would choose its business model and the corresponding pricing strategies in a monopoly market

\section{MODELS}

This paper builds a model for content integrated video websites in monopoly market, and analyzes the choice of business model and pricing strategy. There are three types of participants in the market; they are the video websites acting as the two-sided platform, and audiences and advertisers who are connected by the platform. The audiences could choose to become free users or VIP users according to their preference of the ads and video contents, free users could watch videos with ads with no charge, and VIP users could remove ads and gain add-on utility.

The audiences are receivers of video contents and ads. Model assumes that potential audiences are normalized to 1 , the differentiation of audiences comes from their willing to pay of the contents provided by websites, presented by $\theta$, which means the utility gained by a potential audience from 1 unit product, and researchers assume $\theta \sim[0,1]$. Besides, researchers think the VIP users could get all the utility provided by the platform, depicted as $v$; while the free users could only get part of the total utility $k v(0<k<1)$. For example, audiences could watch video with higher quality $(1080 \mathrm{p}$ $\& 720$ p) by paying subscription fee in iQiYi, while free users can only watch normal ones. Or the VIP users could watch all the authorized edition of movies in LeTV, while free users couldn't. the total utilities gained by the free use and VIP user with the willing to pay as $\theta$ are as below:

$$
\left\{\begin{array}{l}
U_{1}(\theta)=\theta(k v)-\beta m \\
U_{2}(\theta)=\theta v-p
\end{array}\right.
$$

Here, $U_{1}(\theta)$ stands for total utility of a free user with his willing to pay as $\theta . \theta(k v)$ means the basic utility gained by this particular audience from the video contents. Unlike the general two-sided platform, there exist negative cross-group network externalities between the two sides connected by video websites, free users will get hurt by watching ads. The more ads there are the more utilities free users will lose from the platform. $\beta$ stands for the lost utilities from watching one unit ad. If the platform chooses to set $\mathrm{m}$ units of ads, then the total utilities lost by free users would be $\beta m$. $U_{2}(\theta)$ stands for total utility of a VIP user with his willing to pay as $\theta . \theta v$ means the basic utility gained by this particular audience from the video contents, $p$ is the subscription fee charged by the platform, audiences could remove the utility lost by ads.

If the video websites chooses ad-sponsored business model, that is the platform will provide free videos to part or all of the audiences. The more free audiences the platform attracts the more utility the advertiser will get.Researchers Researchers assume there are a number of advertisers in the market, whenever an audience watches ad in the website, these advertisers will get a fixed profit. Therefore, the model assumes that the advertisement fee charged by the platform is in direct proportion to the number of free audiences. Researchers Researchers use $\alpha$ to describe the unit advertising fee charged to the advertisers, and the total profits gained by platform from advertising could be depicted as:

$$
R=\alpha m n_{1}
$$

\section{THE MONOPOLISTIC EQUILIBRIUM}

When there is only one incumbent in the market, this website could choose three different kinds of business models; they are ad-sponsored model, subscription model, and mixed model.

\section{A. Ad-sponsored Model}

In a monopolistic market, when the website chooses ad-sponsored model, all the audiences could connect to the platform with no charge, but the audience must watch corresponding time of ads.

The total utility gained by the audience with this willing to pay as $\theta$ is $U_{1}(\theta)=\theta(k v)-\beta m$, when the utility of this audience is bigger than zero, he will choose to watch videos online. In other word, the point of indifference for the audience to enter the platform or not is $U_{1}(\theta)=0$, and then researchers get $\theta_{1}^{*}=\frac{\beta m}{k v}$. At this time, all the audiences in platform are free users, and the number is $n_{1}^{*}=1-\theta_{1}^{*}=1-\frac{\beta m}{k v}$. Because the platform uses ad-sponsored business model, the profits of the website all come from advertising fee, which is $\pi_{1}=\alpha m n_{1}^{*}=\alpha m\left(1-\frac{\beta m}{k v}\right)$. And $\pi_{1}$ is a function about $\mathrm{m}$, so the monopolistic platform needs to determine the number of $m$ to maximize the profits of itself.Researchers Researchers calculate the best advertisement number for an ad-sponsored platform is $m_{1}^{*}=\frac{k v}{2 \beta}$ through solving the function $\frac{d \pi_{1}}{d m}=0$. And at this time, there are 
$n_{1}^{*}=\frac{1}{2}$ units of free users connected to the platform, and the total profits of the platform are $\pi_{1}^{*}=\frac{\alpha k v}{4 \beta}$.

\section{B. Subscription Model}

As the video copyrights have caught more attention and as the quality of online videos is increased, another type of video websites is coming onto the stage, and the typical one is Netflix. This kind of websites chooses to adopt subscription model. With the help of the Internet, they have moved the off-line DVD rental business to online video watching business; they provide high quality films and TV series with no ads to audiences paying the subscription fee.

The total utility gained by the audience with this willing to pay as $\theta$ through paying the subscription fee $\mathrm{p}$ is $U_{2}(\theta)=\theta v-p$. Compared with the free platform, audiences could get higher utility generally. In a similar way, according to the point of indifference for the audience to enter the platform or not, which is $U_{2}(\theta)=0$, researchers can get $\theta_{2}^{*}=\frac{p}{v}$. At this time, all the audiences in platform are VIP users, and the number is $n_{2}^{*}=1-\theta_{2}^{*}=1-\frac{p}{v}$. Because the platform is based on subscription business model, the profits of the website all come from subscription fee, which is $\pi_{2}=n_{2}^{*} \times p=\left(1-\frac{p}{v}\right) p$. And $\pi_{2}$ is a function about $\mathrm{p}$, so the monopolistic platform needs to determine the number of $p$ to maximize the profits of itself.Researchers Researchers calculate the best pricing charged to audiences should be $p_{2}{ }^{*}=\frac{v}{2}$ through solving the function $\frac{d \pi_{2}}{d p}=0$. And at this time, there are $n_{2}^{*}=\frac{1}{2}$ units of VIP users connected to the platform, and the total profits of the platform are $\pi_{2}^{*}=\frac{v}{4}$.

\section{Mixed Model}

Recently, some video websites combined the former two business models; they provide two products at the same time. The first one is the low quality products with ads embedded, and the second one is paid products with high quality. The total utility gained by the audience with this willing to pay as $\theta$ from free videos is $U_{1}(\theta)=\theta(k v)-\beta m$, and the utility gained by this particular audience from paid videos is $U_{2}(\theta)=\theta v-p$. In this market, the distribution of audiences is as below. According to the indifference point of these two kinds of products $U_{1}(\theta)=U_{2}(\theta)$, researchers can get $\theta_{32}^{*}=\frac{p-\beta m}{v-k v} ;$ and according to the indifference point of connecting to the platform or not $U_{2}(\theta)=0$, researchers can get $\theta_{31}^{*}=\frac{\beta m}{k v}$. Here, the equilibrium of free users count is $n_{31}^{*}=\theta_{32}^{*}-\theta_{31}^{*}=\frac{k p-\beta m}{k v-k^{2} v}$, and that of VIP users count is $n_{32}^{*}=1-\theta_{32}^{*}=1-\frac{p-\beta m}{v-k v}$. Because this platform is providing two products at the same time, the total profits of the platform are the sum of profits from each product. i.e. $\pi_{3}=n_{2} p+\alpha m n_{1}-f$. Here, researchers introduce $f$ as the add-on fixed cost of adopting the mixed model, managing advertisers and audiences simultaneously could make higher cost, that's because these two kinds of participants are quite different, there's little overlap between them, so economics of scale doesn't likely to happen ${ }^{[6]} . \pi_{3}$ is the function $\operatorname{about}(m, p)$, so researchers can get the best pricing strategies of platforms adopting mixed model as:

$$
\left\{\begin{array}{l}
p_{3}{ }^{*}=\frac{2 \alpha \beta v(1-k)}{4 \alpha \beta-2 \alpha \beta k-\alpha^{2} k-\beta^{2} k} \\
m_{3}{ }^{*}=\frac{v k(1-k)(\alpha+\beta)}{4 \alpha \beta-2 \alpha \beta k-\alpha^{2} k-\beta^{2} k}
\end{array}\right.
$$

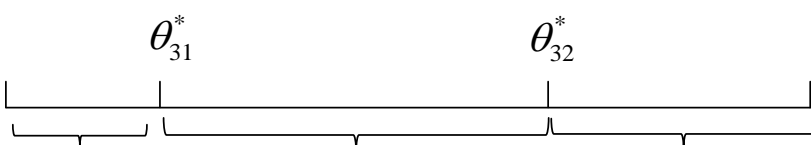

$$
\begin{array}{ccc}
\begin{array}{c}
\text { Audience } \\
\text { would enter }
\end{array} \quad \text { Free users } & \text { VIP users } n_{31}
\end{array}
$$

Fig 1 Distribution of audience under mixed model At this time, the total free users connecting to the platform are $n_{31}^{*}=\frac{\alpha \beta-\beta^{2}}{4 \alpha \beta-2 \alpha \beta k-\alpha^{2} k-\beta^{2} k}$, and the VIP users are $n_{32}^{*}=\frac{2 \alpha \beta-\alpha \beta k-\alpha^{2} k}{4 \alpha \beta-2 \alpha \beta k-\alpha^{2} k-\beta^{2} k}$, the total audiences the platform adopting mixed model attracted are $N_{3}^{*}=n_{31}+n_{32}=\frac{3 \alpha \beta-\alpha \beta k-\alpha^{2} k-\beta^{2}}{4 \alpha \beta-2 \alpha \beta k-\alpha^{2} k-\beta^{2} k}$. And the profits of the platform are:

$$
\pi_{3}^{*}=\frac{\alpha \beta v(1-k)}{4 \alpha \beta-2 \alpha \beta k-\alpha^{2} k-\beta^{2} k}-f .
$$

\section{Conclusion of the Monopolistic Platform}

Researchers put results from section A to section $\mathrm{C}$ into Table 1, and compare the pricing strategies, profits gained by this business model, and the total audiences attracted by the platform.

The necessary requirement of the existing of both free uses and VIP users for mixed model is $\alpha>\beta$, which means the advertising fee of each audience charged to advertisers should be larger than the audiences' utility lost due to 1 unit of advertisement. Under this assumption, total audiences attracted by mixed model are larger than those attracted by pure models are not determined, when 
$2 \beta-k \alpha-k \beta>0$, the mixed model can attracted more compared to the pure models. Consequently, researchers get proposition 1 .

Proposition 1 If the mixed model wants to attract more audiences then the pure models, $2 \beta-k \alpha-k \beta>0$ should be met, which means the addon utilities gained by VIP users through paying the subscription fee are high enough (i.e. $\mathrm{k}$ is small enough).

Proposition 1 means in a monopolistic market, market share gained by the pure model is determined, and the mixed model can differentiate the differences between products provided to free users and VIP users to change its market share. Platforms choosing mixed model must guarantee the utilities from paid contents are high enough to enlarge its market share. In the fact, very few audiences would like to pay for the videos with slightly higher quality, but many of them will pay for the exclusive films.

In Table 1, researchers compare the strategies of ads numbers in pure models and mixed model, and researchers find that if $2 \beta-k \alpha-k \beta>0$, then $m_{11}^{*}>m_{13}^{*}$, and vice versa. And when researchers compare the subscription fee, researchers can find $p_{12}^{*}<p_{13}^{*}$. Consequently, researchers get proposition 2 .

Proposition 2 When the higher add utilities gained by the VIP users through paying the subscription fee, i.e. the smaller the $\mathrm{k}$ is, the fewer ads the video websites can introduce. When $2 \beta-k \alpha-k \beta>0$, the ads in mixed model are fewer than those in the pure model. When there are no differences between the platforms' products, the mixed model can set higher subscription fee.

The conclusion of proposition 2 is very interesting, if the website adopts the mixed model and would like to attract more audiences, it needs to make sure $2 \beta-k \alpha-k \beta>0$, however, under this circumstance, the ads will be fewer than pure model. But compared to pure models, the mixed model can set higher subscription fee. So, in the monopolistic market, if $2 \beta-k \alpha-k \beta>0$, then the transfer from a pure model to a mixed model means subsidy from VIP users to free users.

Finally, researchers compare the platform profits under 3 models, researchers can get $\frac{\pi_{11}^{*}}{\pi_{12}^{*}}=\frac{\alpha k}{\beta}$ and $\pi_{12}^{*}-\pi_{13}^{*}=\frac{(\alpha-\beta)^{2} v}{4\left(4 \alpha \beta-2 \alpha \beta k-\alpha^{2} k-\beta^{2} k\right)}-f>-f \quad . \quad$ If researchers ignore the fixed cost of introducing the mixed model, then the mixed model can bring higher profits. Consequently, researchers get proposition 3 .

Proposition 3 If the add-on utilities gained by VIP users are high enough to satisfy $\beta>\alpha k$, and if researchers don't count f, researchers have $\pi_{13}^{*}>\pi_{12}^{*}>\pi_{11}^{*}$, which means platforms with mixed model can get the highest profits, and those with adsponsored model get the poorest.

\section{CONCLUSIONS}

This paper concentrates on video websites, and builds models to compare the pricing strategies, platforms' profits, and audiences under 3 different business models in the monopolistic market. The main conclusions of this paper are as below:

(1) In a monopolistic market, if the website wants to attract more audiences, it needs to use the mixed model, and must make sure the add-on utilities gained by the VIP users through paying the subscription fee are high enough.

(2) In a monopolistic market, if the website is able to provide contents with high quality, then choosing subscription model would be a better idea; and whether to use mixed model depends on the fixed cost of introducing the mixed model.

TABLE I. PRICING STRATEGIES AND RESULTS OF THREE BUSINESS MODELS

\begin{tabular}{|c|c|c|c|}
\hline Business Model & Pricing Strategy & Platform Profits & \# of Audience \\
\hline Ad-Sponsored & $m_{1}^{*}=\frac{k v}{2 \beta}$ & $\pi_{1}^{*}=\frac{\alpha k v}{4 \beta}$ & $n_{1}^{*}=\frac{1}{2}$ \\
\hline Subscription & $p_{2}^{*}=\frac{v}{2}$ & $\pi_{2}^{*}=\frac{v}{4}$ & $n_{2}^{*}=\frac{1}{2}$ \\
\hline Mixed Model & $m_{3}^{*}=\frac{v k(1-k)(\alpha+\beta)}{4 \alpha \beta-2 \alpha \beta k-\alpha^{2} k-\beta^{2} k}$ & $\pi_{3}^{*}=\frac{\alpha \beta v(1-k)}{4 \alpha \beta-2 \alpha \beta k-\alpha^{2} k-\beta^{2} k}-f$ & $N_{3}^{*}=\frac{3 \alpha \beta-\alpha \beta k-\alpha^{2} k-\beta^{2}}{4 \alpha \beta-2 \alpha \beta k-\alpha^{2} k-\beta^{2} k}$ \\
\hline
\end{tabular}

\section{REFERENCES}

[1] Information on http://www.iresearch.com.cn/report/2262.html

[2] Rochet J C, Tirole J. Platform Competition in Two-sided Markets[J]. Journal of the European Economic Association, 2003, 1(4):990-1029.
[3] XU Li,CHEN Hong-min,PAN Xiao-jun. Research on Price Strategy of Firms in Two-Sided Markets[J]. Journal of Management Sciences in China, 2009, 12(5):10-17.

[4] Casadesus-Masanell R, Llanes G. Mixed Source[J]. Gastón Llanes, 2011, 57(7):1212-1230.

[5] Chao Y, Derdenger T. Mixed Bundling in Two-Sided Markets in the Presence of Installed Base Effects[J]. Management Science 
Journal of the Institute for Operations Research \& the Management Sciences, 2013, 59(8): 1904-1926.

[6] Bourreau M. Mimicking vs. counter-programming strategies for television programs[J]. Information Economics \& Policy, 2003, 15(1):35-54.

[7] Prasad A, Mahajan V, Bronnenberg B. Advertising versus pay-perview in electronic media[J]. International Journal of Research in Marketing, 2003, 20(2):13-30.

[8] Crampes, C., C. Haritchabalet, B. Jullien. Advertising, competition and entry in media industries[J]. Journal of Industrial Economics, 2009, 57(1): 7-31.
[9] Casadesus-Masanell R, Zhu F. Strategies to Fight Ad-Sponsored Rivals[J]. Management Science, 2009, 56(9):1484-1499.

[10] Wu Chang-nan. City Evening Daily: Pricing, Exaggerative Circulation and Regulation - Based on the Theory of Two-sided Market[J]. China Industrial Economics, 2014, 311(2): 109-121.

[11] Zolnowski A, Researchers iss C, Bohmann T. Representing Service Business Models with the Service Business Model Canvas -- The Case of a Mobile Payment Service in the Retail Industry[C]// 2014 47th Hawaii International Conference on System Sciences (HICSS)IEEE Computer Society, 2014:718-727. 